\title{
REMOCIÓN DE ARSÉNICO DEL RÍO SAMA UTILIZANDO LA METODOLOGÍA DE HIERRO CERO VALENTE (Cuenca del Río Sama, Provincia de Tacna)
}

\author{
RIVER ARSENIC REMOVAL SAMA IRON USING ZERO METHODOLOGY \\ VALENTE (Sama River Basin, Province Tacna)
}

\author{
Edgardo Avendaño Cáceres '; Edilberto Mamani López "; Danitza Fabián Fabián ${ }^{\text {s }}$
}

\begin{abstract}
RESUMEN
La tecnología hierro valente cero, aplicada al presente trabajo de investigación, se ha complementado con citrato y radiación solar. La fuente de hierro y citrato fueron la lana de acero comercial y el limón del mercado local de Tacna, los cuales son insumos de bajo costo y de fácil acceso, constituyéndose en una alternativa de solución a nivel domiciliario. La tecnología fue aplicada al agua del río Sama (sector lasYaras). Se planteó un diseño experimental factorial $2^{2}$, más tres puntos centrales con el fin de determinar el efecto de los factores: Cantidad de hierro (g) y limón (gotas), con ese propósito se mantuvo constante el tiempo de radiación solar y el tiempo de sedimentación. Los resultados obtenidos en el área de exploración indicaron que el único factor significativo es la cantidad de hierro, el comportamiento del proceso fue lineal con un $r^{2}=75.63 \%$ y la mayor remoción de arsénico que se logró de las cinco diferentes condiciones experimentales, aplicando una gota de limón y 2 gramos de lana de acero, fue de $98 \%$.
\end{abstract}

Palabras clave:remoción de arsénico, Sama, hierro valente cero.

\section{ABSTRACT}

Zero valent iron technology, applied to the present research work has been supplemented with citrate and solar radiation. The source of iron and citrate were commercial steel wool and lemon Tacna local market, which are low-cost inputs and easily accessible, being an alternative solution to the household level.The technology was applied to river water Sama (theYaras industry). Raised 22 factorial experimental design plus three focal points in order to determine the effect of factors:Total iron ( $g$ ) and lime (drops) that purpose remained constant solar radiation time and the time sedimentation. The results obtained in the scanning area indicated that the only significant factor is the amount of iron, the behavior of the process was linear with $r 2=75.63 \%$ and greater removal of arsenic was achieved by five different experimental conditions, applying a lemon drop and 2 grams of steel wool was $98 \%$

Keywords:arsenic removal, Sama, zero valent iron

\section{INTRODUCCIÓN}

Alrededor de un tercio de arsénico presente en la atmósfera proviene de fuentes naturales, por ejemplo, de los volcanes y el resto, de actividades humanas.

Cuando hay contaminación geológica natural, se pueden encontrar altos niveles de arsénico en el agua de bebida que proviene de pozos profundos, o de ríos y de lagos que se originan en áreas con depósitos de roca volcánica o de minerales ricos en arsénico [2]. En América Latina, por lo menos, cuatro millones de personas en Argentina, Chile, México, El Salvador, Nicaragua, Perú y Bolivia consumen, en forma permanente, agua con niveles de arsénico que ponen en riesgo su salud, como la enfermedad del hidroarsenicismo cuyo avance progresivo culmina en cáncer $[\mathrm{s}]$.

La cuenca del río Sama pertenece al sistema hidrográfico del Pacífico y tiene sus orígenes en las lagunas Calere (Río Cano, 4600 m.s.n.m.), Cotanvilque (Río Jaruma Chico, 4680 m.s.n.m.),

\footnotetext{
'Ingeniero Químico, Doctor en Ciencias y Tecnologías Medioambientales. Facultad de Ingeniería de la UNJBG.

${ }^{2}$ Ingeniero Químico, Magíster en Ciencias Químicas. Facultad de Ingeniería de la UNJBG.

${ }^{3}$ Bachiller en Ingeniería Química. Facultad de Ingeniería de la UNJBG.
} 
Laycacocha (Quebrada Afluente Río Jaruma, 4700 m.s.n.m.) y Caparaja (Quebrada Caparaja, 4900 m.s.n.m.), con un área de drenaje de $4615,65 \mathrm{~km}^{2}$, desde sus nacientes hasta la desembocadura en el Océano Pacífico. La longitud máxima de su recorrido es $128,98 \mathrm{~km}$, aproximadamente.

La cuenca del río Sama presenta una precipitación total mensual promedio que va desde $0,4 \mathrm{~mm}$ (abril) a $8,4 \mathrm{~mm}$ (setiembre), variando algunas de sus características, dependiendo de la estación en que se encuentre [4]. En la provincia de Tarata y zonas aledañas no se desarrolla actividad industrial alguna, siendo la actividad agrícola, ganadera y comercial las principales fuentes de ingreso. Los poblados de los distritos de Sama adquieren el agua para uso primario, líquido elemento que es transportada en cisternas, desde la ciudad de Tacna[5], ya que no cuenta con una planta de tratamiento adecuada para remover arsénico, presente en el río Sama.

Por otro lado, las tecnologías tradicionales para la remoción de arsénico en aguas son caras. De ahí la importancia de aplicar una tecnología existente (transferencia tecnológica) que sea simple, eficiente y de bajo costo para eliminar in situ la sustancia contaminante.

La población de Sama las Yaras cuenta con una población aproximada de 2387 habitantes, según información del INEI (Censo del 2007), siendo su actividad económica principal la agrícola, entre las que destacan el cultivo de alfalfa en un $61,1 \%$, maíz chala en un $20,1 \%$ y en menor cantidad ají, olivo, cebolla, entre otros (según el Plan de cultivo de Riego Campaña 2009-2010) [4]

La metodología utilizada se basa en los resultados de la revisión de recientes estudios para remover arsénico de aguas naturales tal como sigue:

- Remoción de arsénico por oxidación solar en aguas para consumo humano, por Mary Luz Chávez Quijada, Perú (2011), realizada en la región Apurímac, en las aguas del río Iscahuaca - Colcabamba. Las pruebas experimentales se llevaron a cabo con aguas sintéticas, con un $\mathrm{pH}$ promedio de alrededor de 6,7 y $0,2 \mathrm{mg} / \mathrm{L}$ de arsénico, obteniéndose una reducción de $98,5 \%$ del total de arsénico.

- Remoción de arsénico en fuentes de agua natural con Hierro Valente Cero asistido por radiación solar por Lorena Cornejo et al, Chile 2008, realizado en el río Camarones, con concentraciones de arsénico entre 1000 y
$1300 \mathrm{ug} / \mathrm{L}$, logrando determinar las condiciones óptimas $(1,9 \mathrm{~g} / \mathrm{L}$ de lana de acero y una gota de jugo de limón), obteniendo porcentajes de eliminación superiores al 99,5\% y una concentración de arsénico final de $10 \mathrm{ug} / \mathrm{L}$.

- Avances en Tecnologías económicas solares para desinfección, descontaminación y remoción de arsénico en aguas de comunidades rurales de América Latina (Métodos Fh y Raos), Proyecto OEA AE 141, editado por Marta I. Litter y Antonio Jiménez González, Argentina- BrasilChile-México-Perú-Trinidad \& Tobago (2004).

Los grupos pertenecientes a Argentina, Chile, Brasil, México, Perú y Trinidad \& Tobago, propusieron tecnologías de desinfección solar de aguas (DSAUI-SODIS), así como la remoción de arsénico por oxidación solar (RAOS-SORAS) y la fotocatálisis heterogénea $(\mathrm{FH})$. La mayor ventaja de las tecnologías es que son dependientes únicamente de la energía solar y fácilmente aplicables a regiones como las latinoamericanas, con alta incidencia de radiación diurna, es decir, más de 3000 horas de sol en promedio por año, e intensidades luminosas que pueden alcanzar, en algunos casos, $5-6 \mathrm{~kW} / \mathrm{h} / \mathrm{m}^{2}$. Ninguna de ellas requiere equipamiento o desarrollos tecnológicos sofisticados o caros, ni altos costos de energía.

La tecnología RAOS resulta un método de referencia e interés para el presente proyecto de investigación, pues sólo considera a ésta, dejando de lado las otras dos tecnologías expuestas en el proyecto de la OEA.

En la presente investigación se utilizó la tecnología hierro valente cero asistida por radiación solar y citrato. Se basa en el empleo del $\mathrm{Fe}$ metálico para la producción sostenida de hidróxido férrico, capaz de remover compuestos arsenicales presentes en aguas.

La remoción de arsénico por esta técnica generalmente está asociada a procesos de adsorción/co-precipitación de especies de As inorgánico, en especial As (V), al hierro metálico y a sus productos de corrosión (principalmente oxi/hidróxidos)[7], lo que permitió llegar a los límites máximos permitidos por la legislación peruana.

\section{OBJETIVOS}

\section{Objetivo general}

Aplicar la tecnología de hierro cero Valente para la remoción a nivel domiciliar de arsénico del 
río Sama, fuente de abastecimiento de agua del poblado rural del distrito de Sama Las Yaras, provincia de Tacna.

\section{Objetivo específico}

Determinar la influencia de la cantidad de limón y hierro en la aplicación de esta tecnología para remover arsénico de las aguas del río Sama (sector las Yaras), proporcionando una alternativa de solución a nivel domiciliar, mediante una tecnología simple, eficiente, de bajo costo para eliminar in situ la sustancia contaminante en las zonas rurales del distrito de Sama que carecen de agua potable.

\section{MÉTODOS}

\subsection{Población y muestra de estudio}

La muestra de agua estudiada fue representativa. $\mathrm{El}$ punto de muestreo comprende la planta de tratamiento de agua no operativa de Sama las Yaras. Allí se realizaron las pruebas de remoción de arsénico. Inmediatamente terminado el proceso de tratamiento se trasladaron las muestras al laboratorio de Físicoquímica de la Facultad de Ciencias de la Universidad Nacional Jorge Basadre Grohmann de Tacna, para los análisis de arsénico residual.

\subsection{Técnicas, métodos de recolección y análisis de la muestra}

- Calidad de agua. Muestreo: Guía para la preservación y manejo de muestras, Norma Técnica Peruana ISO 5667-3-2001.

- NTP 214.025:1990 AGUA POTABLE. Determinación del pH. Método potenciométrico.

- Determinación de arsénico. Para esta determinación se utilizó el test de arsénico Merckoquant (test semicuantitativo por comparación visual) dicho test tiene un rango de detección de 0,005 a 0,5 mg/L de As.

\subsection{Procesamiento y análisis de datos}

La estrategia que se siguió para el análisis de los datos es como sigue: se utilizó un diseño factorial con dos factores más 3 puntos centrales $\left(2^{2}\right.$ puntos factoriales +3 puntos centrales). Este tipo de diseño permitió hacer un análisis de curvatura (si el comportamiento es lineal o no lineal), dentro de la región experimental para ver el efecto de las variables independientes, sobre la variable respuesta[9]. Los datos fueron analizados utilizando el software Statgraph Centurión versión XV.
Los factores con los cuales se trabajó fueron cantidad de limón (gotas) y cantidad de lana de acero $(\mathrm{g})$ siendo estas las variables independientes; como parámetros constantes se tuvo el tiempo de sedimentación (h) y el tiempo sometido a la radiación solar (h) y como variable respuesta la concentración de arsénico residual $(\mathrm{mg} / \mathrm{L})$.

A continuación se muestra el arreglo experimental planteado:

Tabla N ${ }^{\mathbf{0}}$ 01: Arreglo experimental.

\begin{tabular}{|c|l|c|c|c|}
\hline \multicolumn{2}{|c|}{ Variables } & \multicolumn{3}{|c|}{ Niveles } \\
\cline { 3 - 6 } Independientes & $\begin{array}{l}\mathrm{X}_{1}=\quad \text { Cantidad de } \\
\text { Lim6n (gotas) }\end{array}$ & 1 & 2 & $\mathrm{~s}$ \\
\cline { 2 - 6 } & $\begin{array}{l}\mathrm{X}_{\mathrm{s}}=\text { Cantidad Lana de } \\
\text { Acero }(\mathrm{g})\end{array}$ & 1 & 1,5 & $\mathrm{Q}$ \\
\hline Dependientes & $\begin{array}{l}\mathrm{Y}_{1}=\text { Arsénico residual } \\
(\mathrm{mg} / \mathrm{L})\end{array}$ & \multicolumn{3}{|l}{} \\
\hline
\end{tabular}

Fuente: Propia

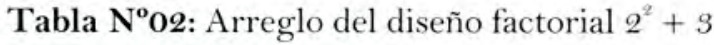
puntos centrales.

\begin{tabular}{|c|c|c|c|c|}
\hline $\mathbf{N}^{\circ}$ & $\mathbf{X}$ & $\mathbf{X}$ & $\begin{array}{c}\text { Limón } \\
\text { (gotas) }\end{array}$ & $\begin{array}{c}\text { Lana } \\
\text { Acero(g) }\end{array}$ \\
\hline 1 & -1 & -1 & 1 & 1 \\
\hline 2 & -1 & +1 & 1 & 2 \\
\hline 3 & +1 & -1 & 3 & 1 \\
\hline 4 & +1 & +1 & 3 & 2 \\
\hline 5 & 0 & 0 & 2 & 1,5 \\
\hline 6 & 0 & 0 & 2 & 1,5 \\
\hline 7 & 0 & 0 & 2 & 1,5 \\
\hline
\end{tabular}

Fuente: Propia

La variable arsénico residual $(\mathrm{mg} / \mathrm{L})$ es la variable respuesta.

\subsection{Procedimiento experimental}

Se tomó como referencia el procedimiento de los diferentes trabajos realizados por Lorena Cornejo et al. [7], [10], [11], [12] con ligeras modificaciones.

El procedimiento consiste en tomar 1 litro de muestra de agua, someterla a exposición solar dentro de una botella transparente PET, en nuestro caso de 3 litros con una inclinación de $20^{\circ}$ en dirección norte. $\mathrm{Al}$ inicio de la reacción se le adicionó a cada botella PET diferentes cantidades de lana de acero y gotas de limón, según lo planteado en el diseño experimental, luego, se procedió a cerrar las botellas y darle agitación manualmente, durante 30 segundos. 
Seguidamente se colocó a exposición solar sin agitación durante 6 horas (mayor radiación solar del día), luego se levantó las botellas y se dejó sedimentar durante 18 horas con el fin de fomentar los procesos de coagulación y sedimentación. Pasadas las 18 horas se procedió al filtrado, usando para tal fin algodón comercial aproximadamente 1,3 gramos por cada muestra.

La muestra filtrada fue sometida al análisis de arsénico y los residuos se depositaron en bolsas plásticas para su disposición final.

\section{RESULTADOS Y DISCUSIÓN}

En la siguiente tabla se muestran algunos parámetros fisicoquímicos, resultado del monitoreo de la calidad de agua, realizados en el 2011 por la Autoridad Nacional del Agua (ANA), mediante la Autoridad Local del Agua (ALA), responsable de las cuencas de Locumba y Sama en Tacna.

Tabla $\mathbf{N}^{\circ}$ 04: Historial de Parámetros fisicoquímicos del Río Sama antes de la captación de la bocatoma la Tranca

\begin{tabular}{|c|c|c|c|}
\hline Parámetros/Fecha & 2011-08-26 & 2011-10-19 & 2011-12-06 \\
\hline $\mathbf{p H}$ & 8,42 & 8,4 & 8,25 \\
\hline $\begin{array}{c}\text { Conductividad } \\
(\boldsymbol{\mu} \mathbf{S} / \mathbf{c m})\end{array}$ & 2270 & 2740 & 2620 \\
\hline $\begin{array}{c}\text { Arsénico Total } \\
(\mathbf{m g} / \mathrm{L})\end{array}$ & 0,2504 & 0,2938 & 0,316 \\
\hline
\end{tabular}

Fuente: Carta $\mathrm{N}^{\circ}$ 113-2012-ANA-AAA-I C-O-ALAL/S

La bocatoma "La Tranca" es el punto de captación del agua del río Sama para el uso de riego y consumo poblacional de Inclán y de las Yaras. La planta de tratamiento se ubica entre la carretera panamericana y la carretera que se deriva hacia el poblado de Buena Vista y de las Yaras.

El pH de la muestra fue de $8,26[1]$ y la concentración inicial de arsénico fue de $0,3 \mathrm{ppm}$. Los reactivos utilizados fueron el hierro que tuvo como fuente la lana de acero comercial y el ácido cítrico cuya fuente fue el limón comprado en la localidad de Tacna.

El tiempo de exposición solar se mantuvo constante e igual a 6 horas, iniciando las pruebas a las 9:00 h y terminando a las 15:00 h. El Servicio Nacional de Meteorología e Hidrología (SENAMHI) de Tacna no cuenta con los sensores para medir la intensidad de radiación solar en la zona, razón por la cual solamente se ha tenido como dato cualitativo el clima que se mantuvo de parcialmente nublado a nublado el día que se realizaron las pruebas experimentales.
Autores como Lorena Cornejo [et al.][7] en pruebas realizadas en aguas del río Camarones (Arica-Chile), menciona que la ausencia de radiación solar retrasa significativamente la remoción de arsénico, y esto podría explicarse considerando que el hierro oxidado forma el complejo citrato-hierro que permanece en solución, evitando la formación de hidróxidos de hierro que, a su vez, adsorben el arsénico. Sus resultados sugieren que para incrementar la eficiencia de remoción es esencial la luz solar, ya que activa la formación de radicales y favorece la oxidación del hierro valente cero, incrementando la velocidad de remoción y eficiencia.

\subsection{Diseño experimental}

Las concentraciones de arsénico residual cuantificadas en las pruebas experimentales se ofrecen en el siguiente gráfico:

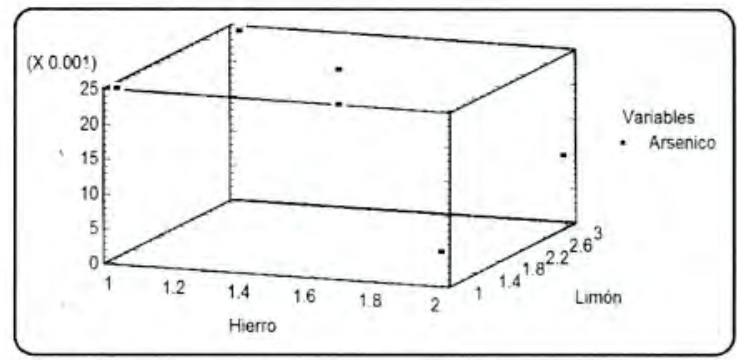

Gráfico $\mathbf{N}^{\circ}$ 01: Resultados Experimentales de las pruebas realizadas utilizando el diseño Factorial

Los resultados del análisis de varianza (ANOVA) mostraron que el único efecto significativo en el área de exploración, con un $95 \%$ de confianza, es la cantidad (g) de hierro. La interacción entre la cantidad de limón y cantidad de hierro, el efecto del limón y la prueba de falta de ajuste resultaron no significativas. Se obtuvo un $r^{2}=75,63 \%$, lo cual nos dice que el modelo lineal ajustado explica el $75,63 \%$ de la variabilidad en la concentración de arsénico residual. El modelo lineal ajustado es el siguiente:

Arsénico Residual (ppm) $=0,0505357$ $0,0025 *$ Limón(gotas) $-0,0225 * \operatorname{Hierro}(\mathrm{g})+$ 0,0025 * Limón (gotas) * Hierro $(\mathrm{g})$

Existen resultados de otros autores que han trabajado con otra calidad de agua [7] y muestran comportamientos no lineales. En la presente investigación se determinó un comportamiento lineal, con $r^{2}=75,63 \%$.

Los datos analizados que nos ocupa son resultados de una única prueba y realizada en campo. Las tempranas precipitaciones pluviales en 
la zona modificaron la calidad del agua y no haciendo posible continuar con la investigación. Se coincide en que el factor cantidad de hierro es el factor principal que favorece a la remoción de arsénico.

\subsection{Remoción de arsénico}

La cantidad de limón y gramos de lana de acero que logró la mayor remoción de arsénico en la presente prueba experimental preliminar (de las 5 condiciones utilizadas en el diseño experimental) fue de 1 gota de limón y 2 gramos de lana de acero, llegando a remover el $98 \%$ de arsénico.

\section{CONCLUSIONES}

El único factor significativo en el área de exploración estudiada fue el factor cantidad de hierro. $\mathrm{El}$ proceso muestra un comportamiento lineal con un $r^{2}=75,63 \%$.

La mayor remoción de arsénico fue de $98 \%$, aplicando 1 gota de limón y 2 gramos de lana de acero.

\section{RECOMENDACIONES}

Considerando que los resultados mostrados en el presente estudio son producto de una única prueba en campo, se recomienda continuar con la investigación. Con el fin de corroborar los resultados (si el comportamiento del proceso es lineal, el porcentaje de remoción se mantiene, entre otros) hacer pruebas en las distintas estaciones del año y plantear un modelo experimental que permita encontrar las condiciones óptimas de remoción.

Es necesario la caracterización química de la lana de acero comercial utilizada, con el fin de asegurar su inocuidad como fuente de aporte de hierro.

Es conveniente también determinar las concentraciones de hierro residual en las muestras, con el fin de asegurar que su valor no sobrepase la normativa vigente.

Por otro lado, como en muchos procesos de remoción de arsénico, se tiene el problema de los residuos generados después del tratamiento (los residuos de algodón con hierro y arsénico retenidos, así como residuos que quedan en las botellas luego del tratamiento). La gestión de estos residuos generados puede ser tema de otra investigación.

\section{REFERENCIAS BIBLIOGRÁFICAS}

[1] Standard Methods for the Examination of Water and Wastewater (1992); APHA; AWWA, and WPCF, 18th ed., Washington, D.C.

[2]J. Ng, A. Gomez Caminero, P. Howe, M. Hughes, E. Kenyon, D.R. Lewis, M. Moore, and by a. Aitio and G. Becking; 2001; Environmental health criteria for arsenic and arsenic compounds.

[3]M. Castro (2004); Arsénico en el agua de bebida de América Latina y su efecto en la salud pública; CEPIS/OPS; Péru.

[4]Estudio Evaluación de Recursos Hídricos Cuenca de los Ríos Locumba y Sama. Ministerio de Agricultura - ALA Locumba Sama. Dirección de Conservación y Planeamiento de Recursos Hídricos. Área de Aguas Superficiales/diciembre(2010).

[5]Dirección de Gestión de la Calidad de los Recursos Hídricos DGCRH/Informe Técnico 197/2011.

[6]M. L. Chávez Quijada (2011); Remoción de Arsénico Por Oxidación Solar En Aguas Para Consumo Humano; Perú. URL: (http://www.scielo.org.pe/scielo.php?pid=S1 810634X2011000400008\&script=sci_arttex.

[7]L.Cornejo, H. Lienqueo, M. Arenas, J. Acarapi, D. Contreras, J. Yáñez, H. D. Mansilla (2008); In field arsenic removal from natural water by zero-valente iron assited by solar radiation; ELSEVIER; Environmental Pollution.

[8]Avances en Tecnologías Económicas Solares para Desinfección, Descontaminación y Remoción De Arsénico En Aguas De Comunidades Rurales De América Latina (Métodos Fh Y RAOS) - Proyecto OEA AE 141 editado por Marta I. Litter y Antonio Jiménez González; Argentina- Brasil-ChileMéxico-Perú-Trinidad \& Tobago (2004).

[9]D. Momgomery (2004); Diseño y Análisis de Experimentos; 2da Edición; LimusaWiley.

[10]L. Cornejo, F. Lara, J. Yáñez, Freer J. (2006); Eliminación de arsénico de aguas naturales asistida por luz solar: efecto de la concentración de hierro y citrato. Chile. 
[11]L. Cornejo, H. D. Mansilla, M. Janet Arenas, M. Flores, V. Flores, L. Figueroa y J. Yañez (2004); Remoción De Arsénico En Aguas Del Rio Camarones, Arica, Chile, Utilizando La Tecnología RAOS Modificada; Chile.

[12].L. Cornejo, J. Yáñez, L. Figueroa, H. Mansilla (2004); Reducción De La Concentración De Arsénico En Agua De Consumo Humano En Zonas Rurales; Chile.http://www.bvsde.ops-oms.org/ bvsair/e/repindex/repi85/vleh/matedu /zonas.pdf

\section{Correspondencia:}

Edgardo Avendaño Cáceres

Ciudad Universitaria fundo "Los Granados" Av. Miraflores s/n Tacna - Perú

Edilberto Mamani López

Ciudad Universitaria fundo "Los Granados" Av. Miraflores s/n Tacna - Perú

Danitza Fabián Fabián

Ciudad Universitaria fundo "Los Granados" Av. Miraflores s/n Tacna - Perú

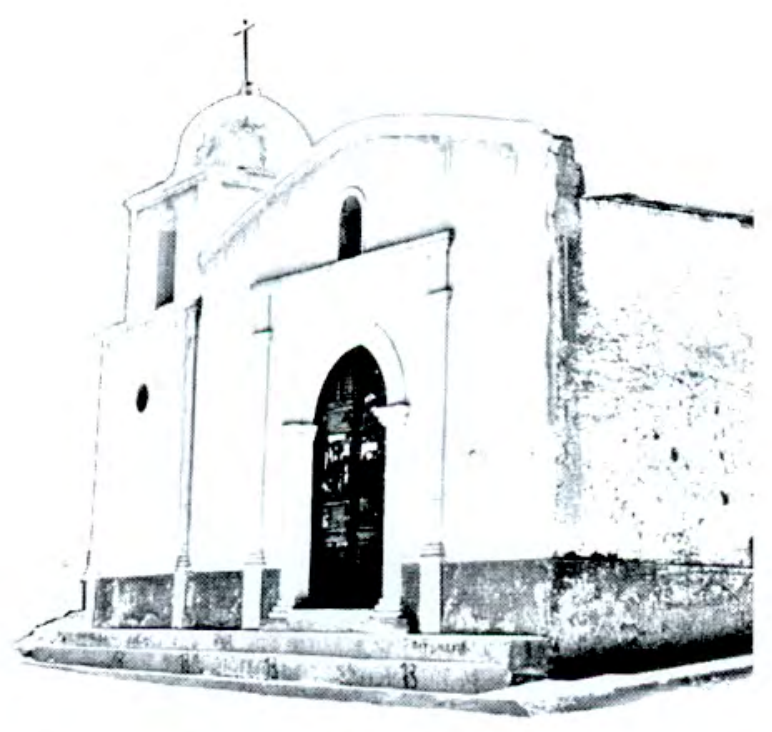

Templo de Estique Pampa 